\title{
Histomorphometric indicators of chicken-broilers spleen of the cobb-500 cross within the species-specific interferon
}

\author{
V.I. Kotarev ${ }^{1}$, E.V. Mikhailov ${ }^{1,}$, N.A. Khokhlova ${ }^{1}$, Yu.A. Chaplygina ${ }^{1}$, A.Yu. Samuilenko ${ }^{2}$, Yu.O. Falkova ${ }^{1}$, and I.I. \\ Misharin $^{1}$
}

${ }^{1}$ All-Russian Research Veterinary Institute of Pathology, Pharmacology and Therapy, 394087 Voronezh, Russia

${ }^{2}$ All-Russian Research and Technology Institute of Biological Industry, 141142 Moscow, Russia

\begin{abstract}
The article presents the data study on the effect of recombinant avian alpha-interferon used by poultry flocks on the histomorphological structure of the spleen of broilers of Cobb 500 cross. The study shows the spleen of the experimental group chickens in a state of physiological maturity, which indicates the formation of the spleen as an immune organ in the early postnatal period becoming an important indicator of determining the state of the organism during its formation and adaptation to the environment.
\end{abstract}

\section{Introduction}

One of the urgent problems of poultry farming is to increase the viability and resistance to poultry diseases. Modern intensive poultry growing technologies are associated with significant physiological stresses. Therefore, the implementation of technological processes requires a detailed approach, taking into account the biological characteristics of the growth and development of birds. The key concepts for the poultry development nowadays and in the near future become efficiency and biosafety. It is possible to obtain high indicators of productivity and product quality only from healthy poultry; therefore, innovations in the field of veterinary science play a special role in modern largescale production [1-3].

The intensive exploitation experience on birds shows the damage mostly caused by diseases occur against a weak immune system. There are different environmental factors affecting the immune system of a bird's body the indoor microclimate, content technology, planting density, size of groups, nutrition level, biological usefulness of compound feeds, etc. [four].

Using the genetic potential of highly productive poultry of domestic and foreign selection, aimed at obtaining maximum productivity, leads to a decrease in the adaptive capacity of the poultry organism to environmental and technological factors characteristic of modern industrial poultry farming. As a result, the response in birds manifests itself in the form of inhibition of the activity of humoral and cellular mechanisms of natural defense, impaired processing of antigenic information, inhibition of antibody synthesis, T-lymphocyte deficiency, and depletion of interferon reserves. As a result of deep chronic metabolic disorders that reduce the protective capabilities of the bird's organism, nonspecific resistance and immunobiological reactivity fall, which creates conditions for increasing susceptibility to opportunistic microorganisms [5].

The immune system is one of the important homeostatic systems of the body, which largely determines the level of animal health and their adaptive capabilities. According to the functional principle the immunocompetent organs of birds are divided into the central (thymus, factory bag) and peripheral (spleen, lacrimal gland). Additionally, the main feature of almost all highly productive bird crosses is reduced resistance and immunity $[6,7]$. The central organs of the immune system are the main site for the development of lymphocytes, and the peripheral ones form the microenvironment in which lymphocytes can interact with antigens and among themselves, creating an immune response.

Moreover, if the mammalian spleen is considered primarily as a blood depot, then the bird spleen does not perform this function, since in the postembryonic period it is only an organ of lymphopoiesis (i.e., exclusively an immunocompetent organ). The central part of the immune system in birds, unlike mammals, is completely separated from the hemocytopoiesis system. So, birds are characterized by topographic and organ isolation of that part of the system that is responsible for the proliferation, maturation and differentiation of both populations of lymphocytes.

As a result of the active spleen participation in immune reactions, the reactive structural changes in this organ, both physiological and pathological, become natural. In the scientific community, there is practically no disagreement on the dynamics of the size of the spleen of chickens during growth: the increase in its mass is directly proportional to the mass of the body in accordance with the growth, and the relative mass of this organ is inversely proportional to the mass of the body. The information obtained suggests that the formation of

Corresponding author: voronezh81@ rambler.ru 
the spleen as a whole is completed by the time the chicks are hatched, i.e. an exceptional role in this process is played by the incubation period of eggs $[8,9]$.

The most common causes of embryo diseases are non-compliance with zoohygienic requirements, violation of the conditions of keeping and feeding, diseases of the reproductive organs, non-infectious and infectious diseases of the parent herd [10]. A bird that has been ill during the embryonic period grows worse and develops, and often is not able to serve as good meat and give qualitative egg productivity in the future [11]. To increase the egg hatchability and increase the safety of chickens for the first days of life, maintaining the immunological reactivity and natural resistance of the birds of the parent herd becomes very important. Currently, recombinant interferons (IFN) are widely used, which are related to cytokines (mediators of immunity) and are represented by a family of proteins with antiviral, immunomodulating, antitumor and other types of activity. Possessing a universally wide spectrum of biological activity, they occupy a leading position among other immunoregulatory drugs.

According to the veterinary medicine, interferons are interesting for their antiviral and immunomodulating properties. Interferons do not belong to foreign compounds, but stay an attribute of the organism itself with a certain degree of species-specificity, i.e., pork interferon is most active in piglets and pigs, bovine in cattle, chicken in chickens, etc. The interferon family of cytokines is involved in the development of natural and acquired immune defenses against various pathogens and pathological stimuli.

The main biological effects of IFN include inhibition of the growth of intracellular and extracellular infectious agents of viral and non-viral nature (chlamydia, rickettsia, protozoa, bacteria); antiproliferative activity; antitumor effect; antimutagenic effect; antitoxic effect; radioprotective effect; suppression or enhancement of antibody production; macrophage stimulation, increased phagocytosis; activation of the cytotoxic effect of sensitized lymphocytes on target cells; activation of natural killer cells; stimulation of histamine release by basophils; induction of prostaglandin E synthesis; stimulation of the production of adhesion factors and molecules.

According to their properties, interferons can claim the role of therapeutic and prophylactic drugs for viral, bacterial and mixed bacterial-viral infections, as well as highly effective immunomodulating and anti-stress agents. They can act as modifiers of the action of other therapeutic drugs, for example, antibiotics, tens of times enhancing the antibacterial effect and neutralizing the negative effect on the immune system $[12,13]$.

\section{The aim of the research}

The aim of the research is to study the histomorphological structure of the young chicken spleen of the Cobb 500 cross breed using alphainterferon of a recombinant avian bird of the parent herd.

\section{Materials and research methods}

Scientific and industrial tests were performed in the conditions of Belaya Ptitsa LLC of Belgorod Region, on a parent flock bird and Cobb 500 cross chickens in two stages to study the effect of recombinant avian alpha interferon on the morphological structure of chicken spleens. At the first stage, experimental and control groups of the parent flock of birds aged 26-28 weeks were formed: three buildings - experimental groups; two cases - control (table 1). Three times interferon with an interval of 72 hours 10 days before the egg laying start at a dose of 1000 IU per kg to body weight according to the manual.

Table 1. Cobb 500 Parent Flock (single housing data)

\begin{tabular}{|c|c|c|c|c|c|c|c|}
\hline \multirow[t]{2}{*}{ Group } & \multirow{2}{*}{ 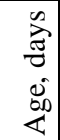 } & \multicolumn{2}{|c|}{ Heads, thousand } & \multicolumn{2}{|c|}{$\begin{array}{l}\text { Live weight } 1 \\
\text { goal, kg }\end{array}$} & \multirow{2}{*}{$\begin{array}{l}\text { Total } \\
\text { live, } \\
\text { tons }\end{array}$} & \multirow{2}{*}{$\begin{array}{l}\text { Water } \\
\text { requirem } \\
\text { ent per } \\
\text { day, tons }\end{array}$} \\
\hline & & chicken & Cock & hicken & cock & & \\
\hline body & 184 & 8.75 & 0.83 & 3.22 & 3.86 & 31.4 & $3-4$ \\
\hline
\end{tabular}

Further from the experimental and control groups the egg was laid for incubation. Next 6 and 20th days, 10 heads of chickens were selected from each group, live weight was determined, then they were removed from the experiment by decapitation and a fragmentary histological autopsy was performed.

Morphometric studies included the determination of the absolute and relative mass of the spleen, which was determined on an analytical balance with an accuracy of $\pm 0.01 \mathrm{~g}$.

For histomorphological studies, spleen samples were fixed in a 10-12\% solution of neutral formalin, dehydrated in an increasing concentration of ethyl alcohol, embedded in paraffin, and serial sections 4-6 $\mu \mathrm{m}$ thick were prepared from paraffin blocks according to the generally accepted method described in $[14,15]$. The general morphological structure of the spleen was studied by staining sections with hematoxylin-eosin. The number and size of lymphatic follicles and the cellular composition of the red pulp were counted on the cross section of the spleen. Morphometry was performed using an ocular measuring grid with a known area $\left(0.0114 \mathrm{~mm}^{2}\right)$ with a magnification of 90 under immersion, as well as on an IBM personal computer using the Meta Vision 1.2 morphological program. The reliability of the data was evaluated using t-student test.

\section{Research results and discussion}

An assessment of body weight and weight of the spleen of young birds of the Cobb 500 cross breed revealed that at the age of 6 days the spleen had a lower weight in the control group than in the experimental group. The mass of the immune organ in the experimental significantly $(\mathrm{P}<0.001)$ increased by $58.8 \%$ relative to the control (table 2). Moreover, differences in body weight of the chickens of the experimental and control groups were unreliable. 
Table 2. Weight of chicken and spleen at 6 days of age

\begin{tabular}{|c|c|c|}
\hline Indicators & The control & Experience \\
\hline Weight of chicken, g & $170.4 \pm 2.26$ & $182.2 \pm 3.507$ \\
\hline $\begin{array}{c}\text { The mass of the } \\
\text { spleen, } \mathrm{g}\end{array}$ & $0.126 \pm 0.0074$ & $0.214 * * * \pm 0.0065$ \\
\hline Weight coefficient & $0.73 \pm 0.03$ & $1.20 \pm 0.10$ \\
\hline
\end{tabular}

During the histological examination of the organ in the control group (A) the spleen was full of blood cells. The venous sinuses of the red pulp are dilated and filled with blood cells. The lymphoid cells in the red pulp and areas of accumulation are detected. The walls of blood vessels (pulpar and trabecular arteries and veins) are thickened in places. The endothelium is swollen, the nuclei are rounded. White pulp in a state of activity. Active lymphoid nodules form.

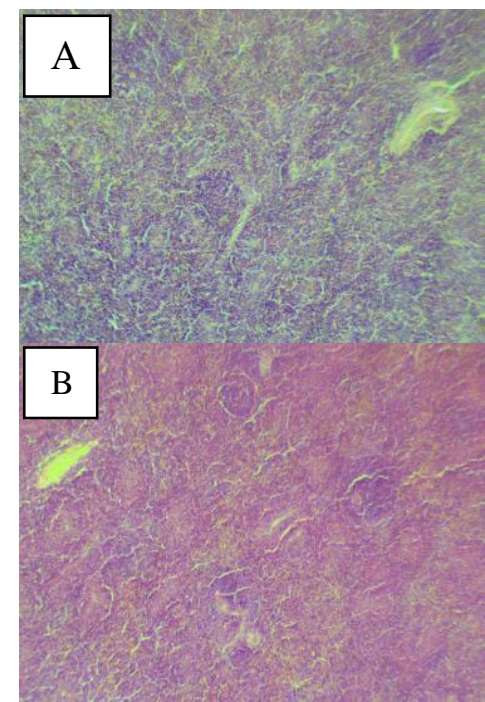

Fig. 1. The architectonics of the spleen of young chickens at the age of 6 days. Hematoxylin-eosin stain

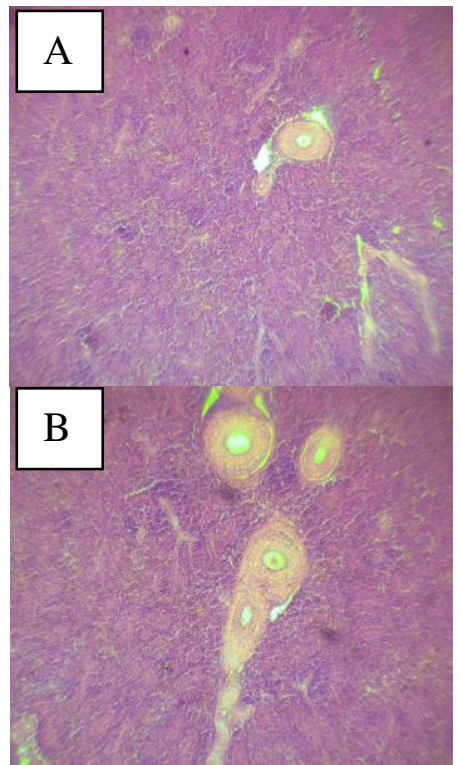

Fig. 2. Structural organization of the spleen of young chickens at the age of 6 days. Hematoxylin-eosin stain
There is an increase in pulpal, trabecular arteries and veins in the experimental group (B). The venous sinuses of the red pulp are dilated, they form blood elements. Multiple, large lymphoid couplings formed around a central artery in the form of a dense ring are revealed in a white pulp. The breeding centers in them are active.

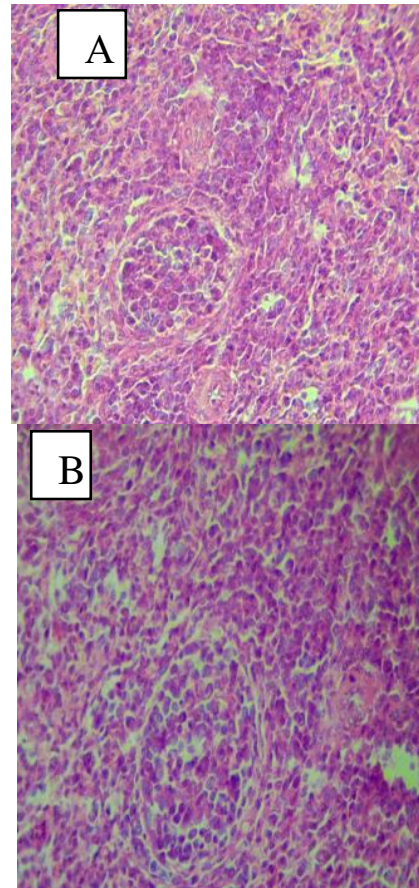

Fig. 3. Architectonics of the spleen of young chickens at the age of 6 days. Hematoxylin-eosin stain

By the age of 21 days, the differences in the average weight of the chickens and the mass of the spleen in the control and experimental groups were not significant (Table 3).

Table 3. Weight of chicken and spleen at 20 days of age

\begin{tabular}{|c|c|c|}
\hline Indicators & The control & Experience \\
\hline $\begin{array}{c}\text { Weight of } \\
\text { chicken, g }\end{array}$ & $910.5 \pm 20.91$ & $890.5 \pm 17.48$ \\
\hline $\begin{array}{c}\text { The mass of the } \\
\text { spleen, g }\end{array}$ & $0.600 \pm 0.058$ & $0.610 \pm 0.035$ \\
\hline $\begin{array}{c}\text { Weight } \\
\text { coefficient }\end{array}$ & $0.68 \pm 0.004$ & $0.71 \pm 0.006$ \\
\hline
\end{tabular}

In this case, an increase in spleen mass occurred in direct proportion to body weight in the period from the 6 th to the 20th day, and the relative mass became inversely proportional to body weight. The information obtained suggests that the formation of the spleen as a whole is completed by the time the chicks are hatched, i.e. an exceptional role in this process is played by the incubation period of eggs [7].

Morphological studies of the spleen from young 20day-old hens in the control group (A) showed the organ parenchyma loaded with red blood cells. Most of the vessels are dilated. The artery walls are thickened, the endothelium is swollen and protrudes into the lumen of the vessel. In the red pulp, the cellular elements are discharged, the white pulp is active and was at a different stage of development. 


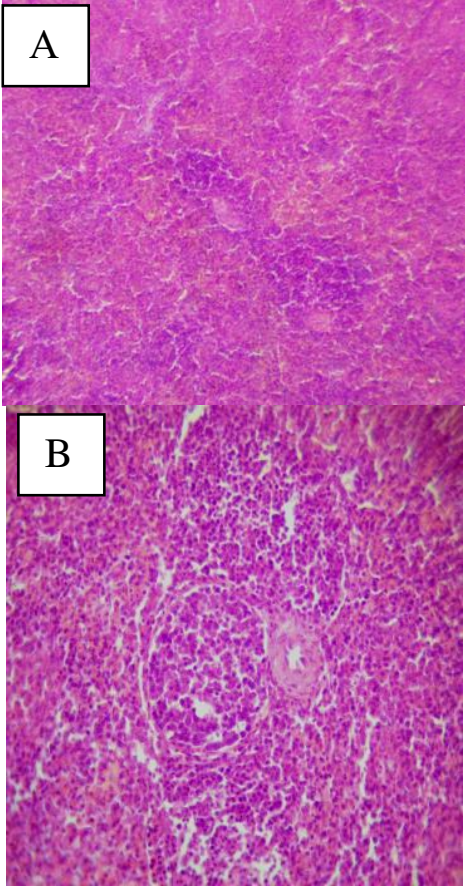

Fig. 4. Structural organization of the spleen of young chickens at the age of 20 days. Hematoxylin-eosin stain.

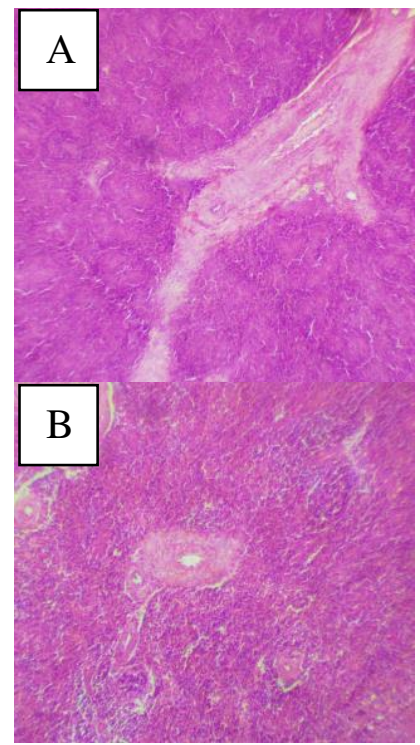

Fig. 5. Structural organization of the spleen of young chickens at the age of 20 days. Hematoxylin-eosin stain

The experimental (B) animals at the age of 21 days had active white. Large couplings are formed. In the Tzone, lymphocytes form a wide zone, lying tightly around the artery. Lymphoid nodules are large, reactive. They express the processes of proliferation and differentiation of cellular elements of lymphoid tissue. Expressed the emergence of new lymphoid couplings. In the splenic cords, near the pulp vessels, foci of plasmaization. Plasma cells at different stages of differentiation. The T-dependent zone of the coupling is wide, the cells form a dense spherical mass around the central artery. In the B-zone processes of proliferation and differentiation. Medium and large lymphoid couplings are well defined, an increase in the total area of germination centers with a clear increase in their diameter has been revealed.

Thus, the obtained morphological data of the immune organ in the early postnatal period is an important indicator of determining the state of the organism during its formation and adaptation in the environment. In the experimental group, at different age periods ( 6 and 20 days), the spleen was in a physiological maturity state, the reproduction centers of the follicles were activated, there was an increase in the area of the white pulp and the activation of germination centers of reproduction, which indicates the formation of the spleen as an immune organ.

\section{Conclusion}

The data obtained during a histomorphological study of the spleen structure of young Cobb 500 chicken crosses using alpha-interferon of a recombinant avian bird of the parent herd indicate that the experimental group at different age periods ( 6 and 20 days) was in a state of physiological maturity, breeding centers follicles were activated, there was an increase in the area of white pulp and activation of germinal reproduction centers. This indicates the formation of the spleen as an immune organ in the early postnatal period and considers an important indicator of determining the state of the organism during its formation and adaptation in the environment.

\section{References}

1. V.I. Fisinin, Integrated development of egg and meat poultry farming in Russia Achievements of science and technology of the agro-industrial complex 10, 9-12 (2008)

2. V.I. Fisinin, Creation of highly productive breeds and crosses of animals and birds Bull. of the Russ. Acad. of Sci. 87(4), 333-336 (2017) DOI: 10.7868 / S0869587317040077

3. V.I. Fisinin, Strategic trends in the development of world and domestic poultry farming: status, challenges, prospects in Mater. of the 19th Int. Conf. "World and Russian trends in the development of poultry farming: realities and challenges of the future" 9-48 (Sergiev Posad, 2018)

4. V.V. Usenko, E.V. Vinogradova, O.V. Koshchaeva, The effect of immunization on the development of internal organs of young egg chickens Scientific J. of KubSAU 105, 1-24 (2015)

5. S.V. Fedotov, M.N. Chernykh, E.A. Kapitonov, The use of immunomodulators for non-specific prophylaxis of mono-mixed infections in chickens Bull. of the Azerbaijan State Agrarian Univer. 5, 97-103 (2012)

6. E.V. Gorshkova, S.V. Kopylova, A.S. Kopylov, E.V. Zaitseva, Comparative macromorphology of spleens of broilers of the cross "Smena-7" and chickens of the cross Highsex brown Vestnik of Bryansk State Agricult. Acad. 2, 27-30 (2014) 
7. V.A. Belyaev, E.E. Epimakhova, D.A. Zinchenko, The influence of the age of the parent herd on the immune organs of broiler chickens Collection of sci. works of VNIIOK 9, 259-263 (2016)

8. A.G. Koshchaev, E.V. Vinogradova, V.V. Usenko, R.D. Litvinov, Morphological features of the spleen of growing chickens under conditions of minimal antigenic load Proceedings of KSAVM named after N.E. Bauman, 3, 39-43 (2016)

9. S.B. Seleznev, V.V. Pronin, M.S. Dyumin, S.P. Fisenko, Structural features of the immune system of birds Russ. Veter. J. 3, 28-30 (2016)

10. V.I. Fisinin, A.Sh. Kavtarashvili, Sh.A. Iman ghouls, Biological basis for increasing the efficiency of production of chicken eggs (Sergiev Posad, 1999)

11. L.V. Sycheva, The influence of the feed additive "Omego-Stip" on the quality indicators of broiler meat Achievements of sci. and technol. of the agroindustr. Complex 5, 53-55 (2013)

12. A.N. Narovlyansky, F.I. Ershov, A.L. Gunzburg, Interferons: promising areas of research Immunology 3, 168-172 (2013)

13. V.A. Prokulevich, M.I. Potapovich, Interferonbased veterinary preparations Bull. of BSU. Ser. 2, 3, 51-55 (2011)

14. G.V. Merkulov, Course of histopathological technology (Medicine, Moscow, 1969)

15. S.M. Suleymanov, A.V. Grebenshchikov, E.V. Mikhailov, I.S. Tolkachev et al., Methods of morphological studies 2rd ed. (All-Russian Research Veterinary Institute of Pathology, Pharmacology and Therapy, Voronezh, 2007) 